\title{
Spin Coated Plasmonic Nanoparticle Interfaces for Photocurrent Enhancement in Thin Film Si Solar Cells
}

\author{
Miriam Israelowitz, ${ }^{1}$ Jennifer Amey, ${ }^{2}$ Tao Cong, ${ }^{2}$ and Radhakrishna Sureshkumar ${ }^{2}$ \\ ${ }^{1}$ Department of Electrical Engineering, Syracuse University, Syracuse, NY 13244, USA \\ ${ }^{2}$ Department of Biomedical and Chemical Engineering, Syracuse, NY 13244, USA
}

Correspondence should be addressed to Radhakrishna Sureshkumar; rsureshk@syr.edu

Received 15 November 2013; Accepted 10 December 2013; Published 9 February 2014

Academic Editor: Hui Xia

Copyright (C) 2014 Miriam Israelowitz et al. This is an open access article distributed under the Creative Commons Attribution License, which permits unrestricted use, distribution, and reproduction in any medium, provided the original work is properly cited.

\begin{abstract}
Nanoparticle (NP) arrays of noble metals strongly absorb light in the visible to infrared wavelengths through resonant interactions between the incident electromagnetic field and the metal's free electron plasma. Such plasmonic interfaces enhance light absorption and photocurrent in solar cells. We report a cost-effective and scalable room temperature/pressure spin-coating route to fabricate broadband plasmonic interfaces consisting of silver NPs. The NP interface yields photocurrent enhancement (PE) in thin film silicon devices by up to $200 \%$ which is significantly greater than previously reported values. For coatings produced from Ag nanoink containing particles with average diameter of $40 \mathrm{~nm}$, an optimal NP surface coverage $\phi$ of $7 \%$ is observed. Scanning electron microscopy of interface morphologies revealed that for low $\phi$, particles are well separated, resulting in broadband PE. At higher $\phi$, formation of particle strings and clusters causes red-shifting of the PE peak and a narrower spectral response.
\end{abstract}

\section{Introduction}

Thin film silicon ( $\mathrm{Si}$ ) solar cells reduce material cost of photovoltaic (PV) systems and offer a means to more affordable renewable energy production. Thin film silicon PVs have an average cell thickness of $300-500 \mathrm{~nm}$ as compared to 200$500 \mu \mathrm{m}$ for bulk crystalline silicon (c-Si) ones [1]. However, the major disadvantage of thin film Si PVs is the relatively low efficiency of light to power conversion due to the low light absorption rate of $\mathrm{Si}$ and the reduced optical path length of the thin film. Currently, the best research cell efficiencies recorded for thin film single junction amorphous $\mathrm{Si}$ are $13.4 \%$ compared with bulk c-Si of $27.6 \%$ [2]. Therefore, in order to realize the potential of thin film Si PVs, efficient broadband light trapping technologies need to be integrated into the device design.

The use of plasmonic interfaces offers much promise for increasing the light trapping efficiency of thin film PVs. Plasmonics play a large role in determining the optical properties of metal nanoparticles (NPs). Specifically, the phenomenon of localized surface plasmon resonance (LSPR) which refers to the strong coupling of the incident electromagnetic field and the free electron plasma of the metal determines the frequencies at which metallic NPs strongly scatter light. Hence, metallic NP coatings can be tailored to create plasmonic interfaces on $\mathrm{Si}$ thin film PVs to efficiently scatter light at large angles into the underlying semiconducting layer and increase the optical path length. There are two fundamental mechanisms proposed to explain how the NPs lengthen the optical path in thin film PVs. First, when the NP diameter is significantly smaller than the wavelength of the incident light, the optical field characteristics of the particle can be approximated as a point dipole, which can reradiate the light acting as a powerful scattering element [3, 4]. For a metal NP with complex permittivity $\varepsilon_{p}$ embedded in a homogenous medium with permittivity $\varepsilon_{m}$, depending on the particle volume $V$ and the incident wavelength $\lambda$, the effective scattering cross-section can be much larger than the physical cross-section of the particle. The scattering and absorption cross-sections, $C_{\text {scat }}$ and $C_{\text {abs }}$, respectively, are given by $[3,4]$ :

$$
C_{\text {scat }}=\frac{1}{6 \pi}\left(\frac{2 \pi}{\lambda}\right)^{4}|\alpha|^{2}, \quad C_{\mathrm{abs}}=\frac{2 \pi}{\lambda} \operatorname{Im}[\alpha],
$$


where

$$
\alpha=3 V\left[\frac{\varepsilon_{P} / \varepsilon_{m}-1}{\varepsilon_{P} / \varepsilon_{m}+2}\right]
$$

is the polarizability of the particle. The surface plasmon resonance occurs when $\varepsilon_{p}=-2 \varepsilon_{m}$ for which the scattering cross-sections increase in size dramatically [3]. Second, a dipole nearby a planar interface of higher optical density can strongly couple evanescent waves otherwise lost, resulting in near field light concentration [5]. A dipole can be modeled as the superposition of its propagating and evanescent waves $[5,6]$. Propagating waves transmitted into the dielectric will do so at angles less than the critical angle of the dielectric boundary. Conversely, evanescent waves transmitted into a dielectric will propagate at angles greater than the critical angle [7]. The power of the radiation from evanescent sources decreases exponentially depending on the distance of the dipole from the surface. The scattering of light by a NP shows a symmetric radiation pattern when embedded in a homogenous material. However, this pattern changes when brought into the vicinity of a dielectric surface, in which the light will scatter primarily into the dielectric of larger permittivity [3]. The fraction of incident light scattered into the substrate using a dipole near a dielectric interface could be as large as $96 \%$ [3].

Light trapping and photocurrent enhancement (PE) by plasmonic interfaces have been well documented in the literature. There have been several methods reported in the literature to create plasmonic interfaces, such as electron beam lithography [8], thermal evaporation [9], nanoimprinting lithography [10], and ns and fs pulsed laser patterning of ultrathin films [11-13]. The most common plasmonic interfaces used for light trapping consist of NP islands formed through thermal evaporation of a metal film followed by annealing $[8,14,15]$. However, this method creates typically a limited range of particle sizes and shapes. Conversely, synthesis of plasmonic NPs using colloid chemistry techniques offers much greater control over particle size, shape, monodispersity, and passivating shell layers $[16,17]$. Subsequently, manufacturing of plasmonic interfaces from NP suspensions using wet chemistry methods could offer robust and controllable means for tailoring plasmonic interfaces with desired optical properties $[18,19]$. Herein, we report a costeffective and scalable room temperature/pressure nanomanufacturing process based on a spin-coating technique to create broadband light trapping plasmonic interfaces on silicon-oninsulator (SOI) devices. Such interfaces are shown to facilitate strong PE of the SOI device up to $200 \%$.

\section{Methods}

2.1. General. All chemicals were bought from Aldrich and used as received unless otherwise stated. A tabletop centrifuge (Eppendorf 5418) was used for NP purification and isolation. Ocean Optics UV/Vis spectrometer (USB4000UV-VIS) was used to characterize NP absorption spectra. Particle size was measured using dynamic light scattering (DLS) on a Malvern Zetasizer Nano Series instrument utilizing $173^{\circ}$ backscatter. SOI wafers were purchased from
University Wafer. The device contacts were fabricated using a thermal evaporator (CVC SC4500) and SEM images (Zeiss Ultra 55) were taken at the Cornell NanoScale Science and Technology Facility (CNF). NPs were applied to SOI devices using a Laurell Technologies Corporation Spin-coater (WS650Mz-23NPP).

2.2. Preparation of SOI Devices. SOI cells were used to measure the NP effects on photocurrent. SOI cells are simple devices that have an accessible open surface that enabled easy monolayer coverage of NPs close to the c-Si active layer within a few nanometers. The wafer was cleaned using the RCA process and metal finger contacts were deposited by thermal evaporation. A mask was used to add $1 \mathrm{~mm} \times$ $10 \mathrm{~mm}$ aluminum contact pairs with a finger distance of $1 \mathrm{~mm}$ apart using thermal vapor deposition and the contacts were deposited to a thickness of $35 \mathrm{~nm}$. The wafer was allowed to rest for a minimum of four hours in standard atmospheric conditions to let the native $\mathrm{SiO}_{2}$ regrow on the top active layer. The wafer was a bonded c-Si n-type wafer, doped with Phosphorous and a resistivity of $1-10 \Omega \cdot \mathrm{cm}$. In true thin film a-Si solar cells the a-Si active layer is thinner than many commercially available SOI wafers; however SOI wafers are thin enough to approximate the actual active device thickness. In our case the c-Si SOI active layer was $\sim 3-4 \mathrm{x}$ thicker than the active layer of standard thin film solar cells.

2.3. Preparation of Silver Ink Solutions. A series of ethanol solutions containing ethylene glycol capped Ag NPs (10, $5,1,0.5,0.2,0.1,0.05,0.01 \% \mathrm{w} / \mathrm{v})$ were prepared from a stock solution of the exact same materials $(20 \% \mathrm{w} / \mathrm{v})$. DLS measurements showed an average particle size; $d=40 \pm$ $1 \mathrm{~nm}$. Ethanol was chosen to dilute the Ag NPs because of the relatively high volatility of the liquid and it would dry off of a surface quickly after being deposited without leaving residues that may block light and suppress current.

2.4. Synthesis of Glucose Capped Silver NPs. Size controlled NPs may be produced through chemical synthesis by varying the $\mathrm{pH}$ of solution during production [20]. An aqueous solution of colloidal Ag particles stabilized by glucose was prepared by adding ammonia $\left(0.02 \mathrm{~mol} \mathrm{~L}^{-1}\right)$ to silver nitrate solution $\left(10^{-3} \mathrm{~mol} \mathrm{~L}^{-1}\right)$. Thereafter, a solution of glucose $\left(0.01 \mathrm{~mol} \mathrm{~L}^{-1}\right)$ was added and the reaction was stirred vigorously for 2 minutes. DLS measurements showed an average NP size; $d=31 \pm 1 \mathrm{~nm}$. In a separate experiment to make larger particles, the procedure was repeated as stated above, but the ammonia concentration was increased to $0.1 \mathrm{~mol} \mathrm{~L}^{-1}$ and the reaction incubation time was increased to 12 minutes. DLS measurements showed an average NP size, $d=69 \pm 1 \mathrm{~nm}$. The particles were cleaned by centrifuging the solution for ten minutes to remove excess glucose.

2.5. Preparation of Plasmonic Interfaces. In all experiments, the bare SOI device was placed into a spin-coater and vacuum applied. The speed of the spin-coater was ramped from $0 \mathrm{rpm}$ to $8000 \mathrm{rpm}$ over a time period of 0.5 minutes. During the spinning speeds of $2000-8000 \mathrm{rpm}$, a solution of colloidal 


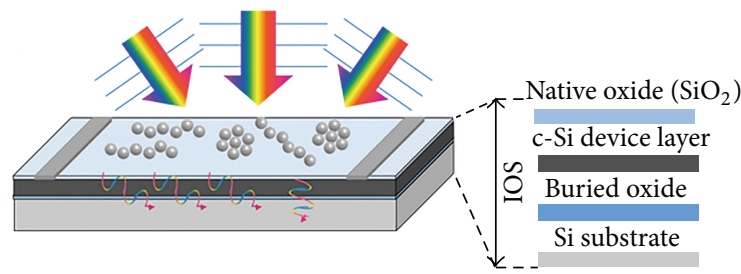

(a)

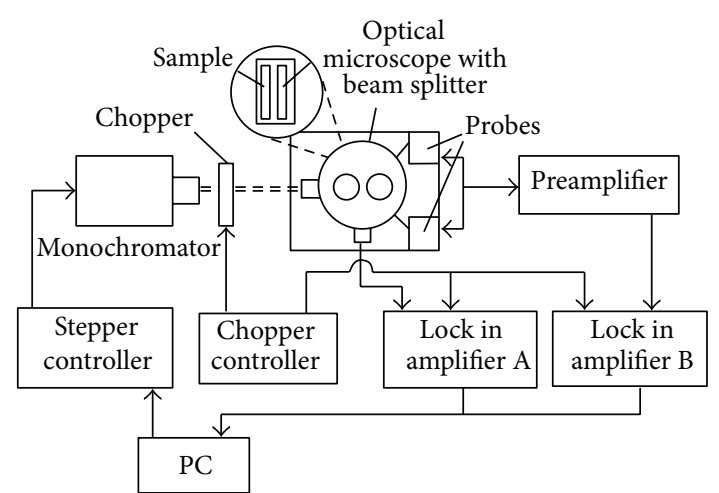

(b)

FIgURE 1: (a) Schematic of the SOI device with nanoparticles. The aluminum contacts were deposited using thermal evaporation. The nanoparticles were deposited through spin-coating. (b) Experimental current collector schematic.

particles $(300 \mu \mathrm{L})$ was deposited onto the bare SOI device using an eppendorf micropipette, one drop at a time in a continual fashion. After the total volume of solution was deposited, the coated SOI device was left to spin at $8000 \mathrm{rpm}$ and dried under vacuum for an additional 29.5 minutes.

2.6. Photocurrent Measurements. The SOI device was a metal-semiconductor-metal (MSM) photodetector that has a single doped n-type c-Si layer with lateral Schottky barrier contacts as shown in Figure 1(a), similar to the experimental setup reported by Pillai et al. [14]. An electrical gradient was applied to the contacts to induce the "on" state of the diode. The MSM photodetector is favorable for photocurrent measurement experiments because the photocurrent generated is linearly proportional to the optical power of the incident light [21-23]. Photocurrent is a key variable in determining both the open circuit voltage and the short circuit current of a solar cell. Improving the photocurrent generated would translate into greater solar cell efficiency. A tunable step-motor monochromator irradiated the light at discrete wavelengths from 400 to $1000 \mathrm{~nm}$ as seen in Figure 1(b). The light source was a halogen lamp under non-AM 1.5 standard conditions. The irradiance larger than $1000 \mathrm{~nm}$ had very low power and thus was difficult to discern the signal from noise. The beam was directed to an optical microscope and focused on the sample and reference using an internal beam splitter. The induced photocurrent was measured via probes using a preamplifier across the contacts. The signal was extracted using a lock in amplifier and sent to the control computer. The short circuit current was recorded as a function of wavelength over the contact area using an analysis computer. The photocurrent response for the SOI device was recorded several times on each sample at different points on the $\mathrm{p}-\mathrm{n}$ metal contacts and averaged to get the overall photocurrent response. The $\mathrm{PE}$ is defined as the ratio of the difference between the photocurrent generated after and before NP deposition to that before deposition expressed in terms of a percentage $[14,15]$. Therefore, the uncoated SOI device was first measured to get the base photocurrent. Immediately after the bare surface measurement, the Ag nanoink or synthesized particle solutions were deposited via spin-coating and dried at ambient conditions.

\section{Results}

3.1. Silver Nanoink. The samples used to measure the PE caused by the silver (Ag) nanoink coating were prepared on SOI wafers with a $2 \mu \mathrm{m}$ n-type crystalline silicon (c-Si) active layer by spin coating. A schematic depicting the structure of the SOI device is shown in Figure 1(a). The structure of the wafer consisted of a neutral Si substrate of $625 \mu \mathrm{m}$ thickness, a buried oxide layer of $1 \mu \mathrm{m}$ thickness, and a top c-Si ntype layer of $2 \mu \mathrm{m}$ thickness. The samples were irradiated at discrete wavelengths and the resulting photocurrent was measured and recorded as shown in Figure 1(b). The absorption range for the $2 \mu \mathrm{m}$ thick SOI device was 400-1000 nm. All samples showed the same shifted interference fringe pattern at wavelengths larger than $700 \mathrm{~nm}$ superimposed on any surface plasmon resonance peaks. Interference patterns appear in the raw data because of transmission and reflection interference within the thin c-Si active layer. These fringes are phase shifted by the silver particles so that the photocurrent responses show the constructive or destructive PE patterns $[4,24]$.

Figure 2 shows the relative photocurrent enhancement responses for eight different Ag NP concentrations. For Ag $\mathrm{NP}$ ink concentrations in the range of $0.01-0.1 \% \mathrm{w} / \mathrm{v}$, the resulting PE curves show a significant broadband response. According to (1) and (2), the LSPR peak of silver in air occurs at $\lambda=375 \mathrm{~nm}$ which is outside the range of operation of our device. Two small peaks of increasing strength with increasing concentration appear at $\lambda \approx 475$ and $760 \mathrm{~nm}$ because of the long range dipole-dipole interactions near a dielectric surface $[25,26]$. As the particle coverage becomes denser, the distance between particles decreases and long range interactions increase. For Ag NP ink concentrations in the range of $1-10 \% \mathrm{w} / \mathrm{v}$, the recorded $\mathrm{PE}$ curves show substantial red-shifting. For $\lambda$ between 400 and $525 \mathrm{~nm}, \mathrm{PE}$ decreases, but it quickly increases for $\lambda>525 \mathrm{~nm}$. Because the curve cannot be shifted past $1100 \mathrm{~nm}$ which corresponds 


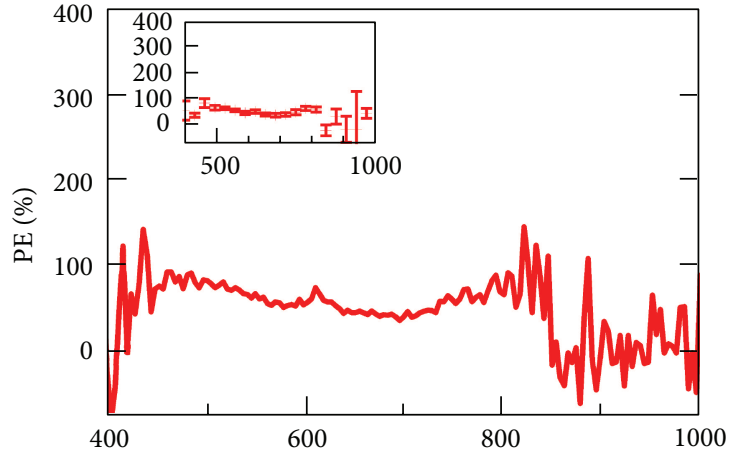

(a)

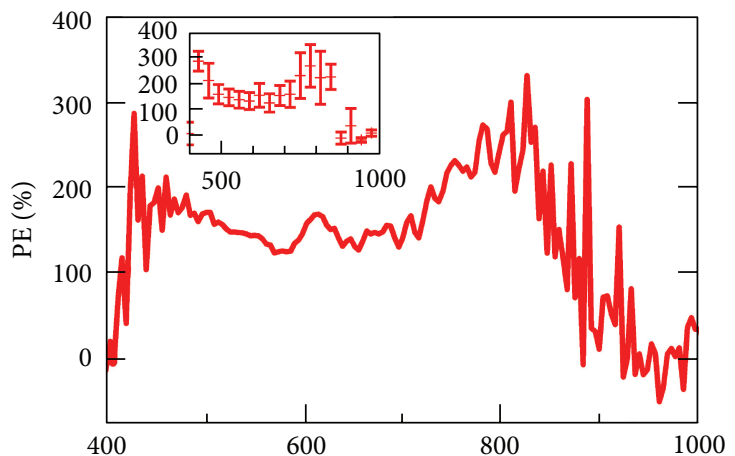

(c)

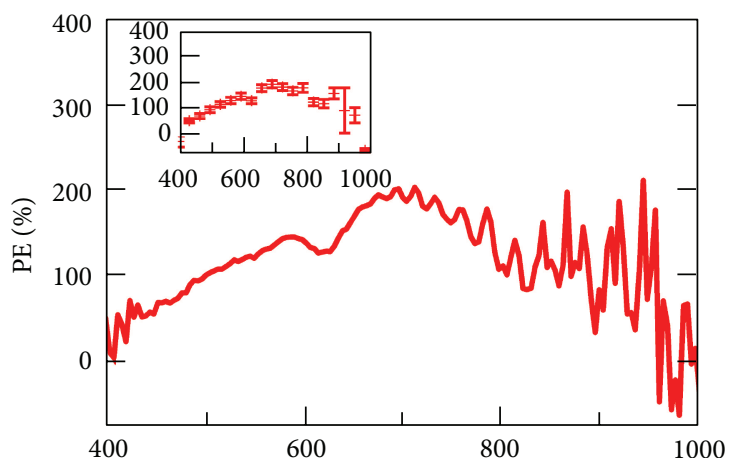

(e)

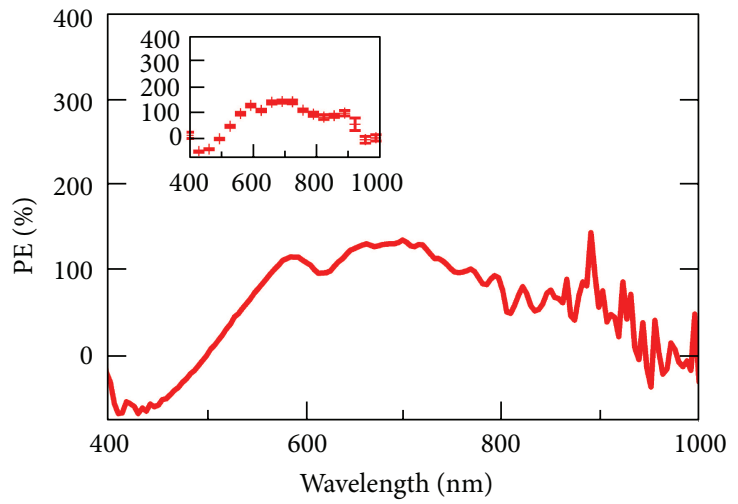

(g)

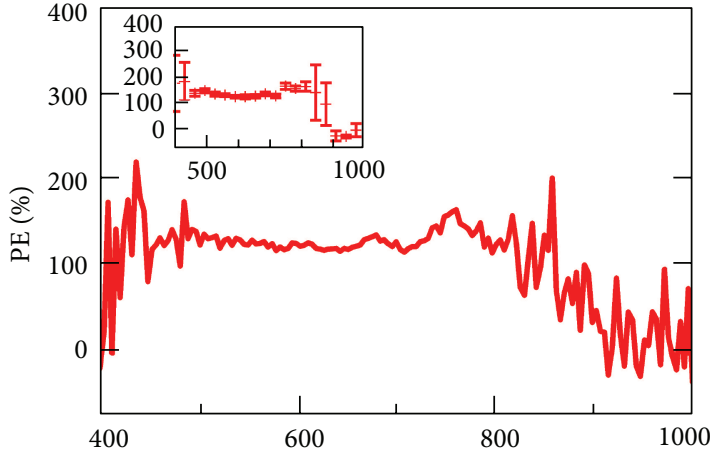

(b)

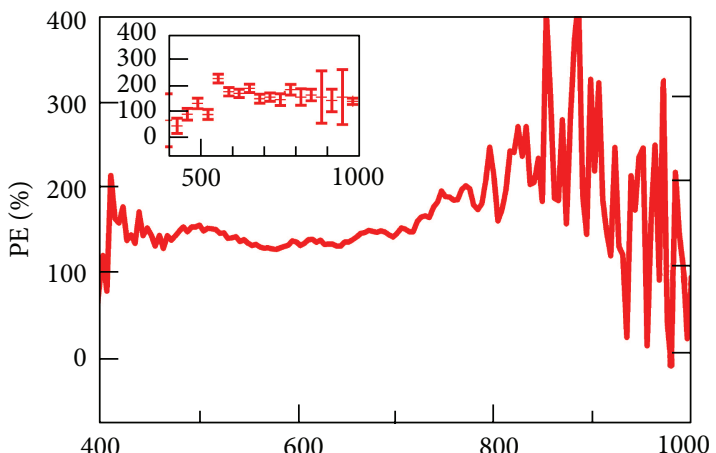

(d)

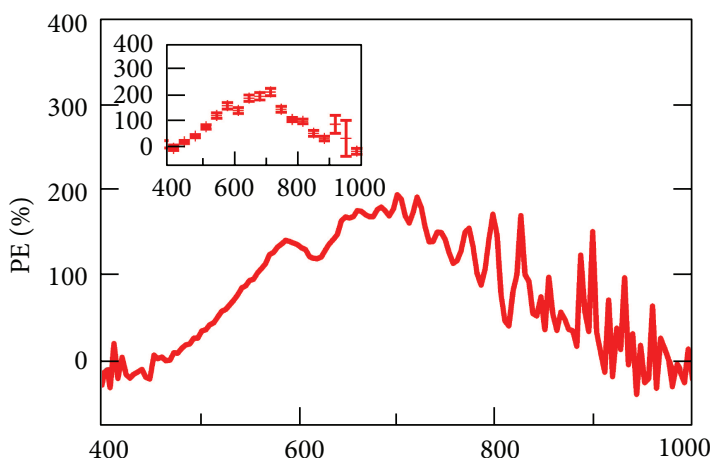

(f)

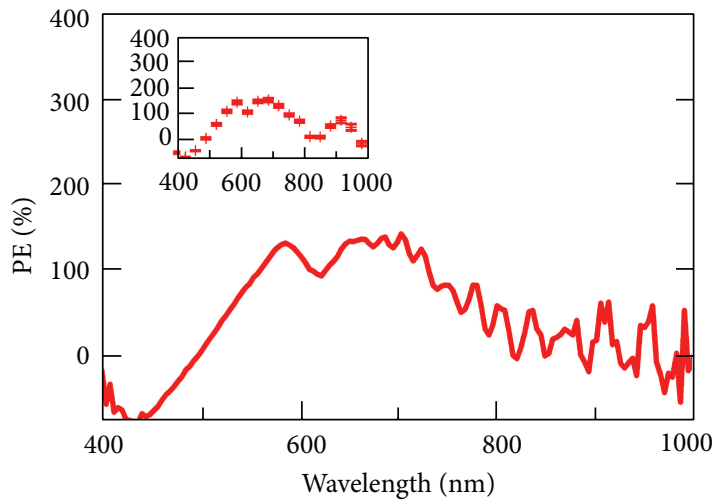

(h)

Figure 2: PE response of (a) $0.01 \%$, (b) $0.05 \%$, (c) $0.1 \%$, (d) $0.2 \%$, (e) $0.5 \%$, (f) $1 \%$, (g) $5 \%$, and (h) $10 \%$ w/v. Ag nano ink coatings. The graphic inset error bars show a sampling of the standard error for the measurements. 
to the bandgap of c-Si, red-shifting of the LSPR results in the observed narrowing of the spectrum. The greatest overall PE is observed for coatings fabricated from a nano-ink solution with a concentration $\rho=0.1 \% \mathrm{w} / \mathrm{v}$ of $\mathrm{Ag}$, for which the spectrum exhibits the greatest overall broadband response. The overall $P E$ is defined as the ratio of the difference between the integrals of the photocurrent response curve after and before NP deposition to that before NP deposition in terms of a percentage. PE increases with increasing $\rho$ until up to $\rho=0.1 \% \mathrm{w} / \mathrm{v}$. In this concentration regime, the coating consists of well-separated particles; see Figure 5 and Section 4. Therefore, the total scattered flux and hence the PE, can be expected to increase linearly with surface coverage. This is corroborated by the observed approximately linear increase in $\mathrm{PE}$ with increasing $\rho$ for $\rho \leq 0.1 \% \mathrm{w} / \mathrm{v}$, as shown in Figure 3. Moreover, for $\rho$ ranging between 0.1 and $0.5 \% \mathrm{w} / \mathrm{v}$, the PE is relatively insensitive to $\rho$ and a nearplateau region can be seen. Further increase in $\rho$ results in a substantial reduction in PE. This observed behavior in $\mathrm{PE}$ can be understood based on the changes in the coating morphology as a function of $\rho$ : see Section 4 .

3.2. Effect of Particle Size: Glucose Capped Silver NPs. It is difficult to obtain exquisite control of particle size in commercially available Ag nano-ink solutions. Hence, in order to decipher the role of particle size on PE, we synthesized spherical Ag NPs encapsulated within a glucose shell following the procedure outlined in Section 2. Two separate batches of Ag NPs were synthesised with average diameters $d$ of $31 \pm 1 \mathrm{~nm}$ and $69 \pm 1 \mathrm{~nm}$, as determined from dynamic light scattering (DLS) measurements. The smaller particle batch had a glucose shell of $\sim 1 \mathrm{~nm}$ whereas the larger particle batch had $\sim 20 \mathrm{~nm}$ glucose shell. The particle concentration was $0.002 \% \mathrm{w} / \mathrm{v}$. The SOI device used for photocurrent measurements had a $1.5 \mu \mathrm{m}$ thick c-Si active layer. The particles were deposited via spin-coating and the photocurrent response was measured in the exact same manner as for the Ag nano ink coatings. Figure 4 shows the photocurrent response for (a) $31 \mathrm{~nm}$ and (b) $69 \mathrm{~nm}$ particles. The overall PE for the $31 \mathrm{~nm}$ particles was $49 \pm 1 \%$. The PE obtained for $d=69 \mathrm{~nm}$ was $199 \pm 3 \%$. This large PE can be explained based on the quadratic increase in the scattering cross-section with increasing particle volume. The albedo of particles at $500 \mathrm{~nm}$ wavelength is shown in Figure 4(c) (blue line). The albedo $A$ is defined as $C_{\text {scat }} /\left(C_{\text {scat }}+C_{\text {abs }}\right)$. The normalized PE responses of the two particle sizes tested are shown in red circles in Figure 4(c) and follow the same trend as $A$, suggesting that scattering from the dipoles (particles) is the key mechanism of PE in dilute systems.

\section{Discussion}

In this section, we correlate the percentage surface coverage $\phi$ and surface morphology of the plasmonic interfaces fabricated by spin-coating to the particle concentration $\rho$ in the feed solution. Further, we utilize theoretical models for the optical response of particle strings and clusters to understand the observed PE-morphology relationship [27,

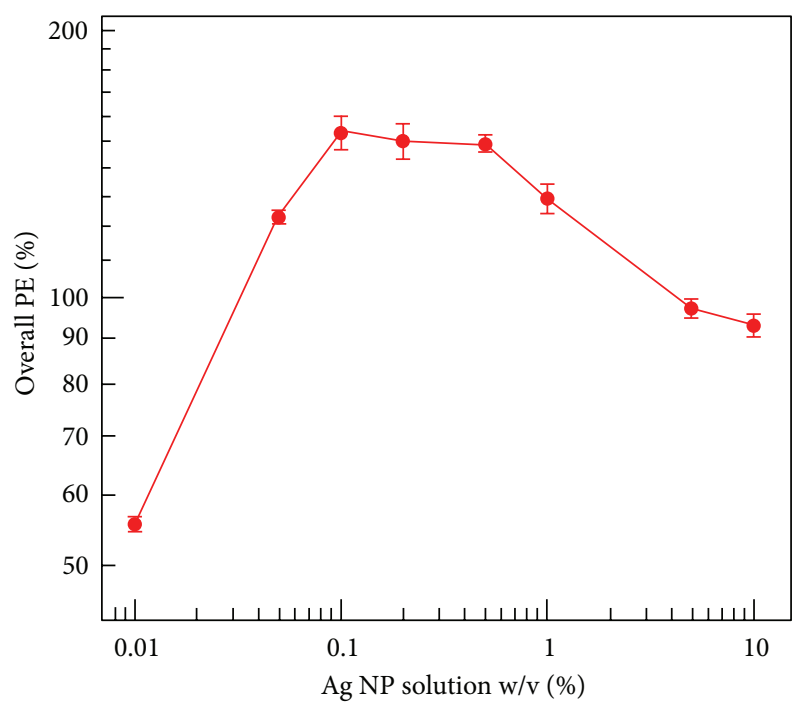

FIGURE 3: Overall PE versus silver concentration in solution. Error bars show the standard error for the measurements.

28]. The effectiveness of NP-mediated scattering mechanisms on PE depends on a number of factors such as the NP size, shape, material, particle-substrate distance, and surface morphology/coverage.

The morphological features and $\phi$ of the coatings were determined by analyzing the scanning electron microscope (SEM) images of the interface. Image J software was used to isolate the particles and determine their size and area coverage. Figure 5(a) shows the SEM images for coatings created from Ag nano-ink solutions with $\rho=0.1,1,5$, and $10 \% \mathrm{w} / \mathrm{v}$. As seen in Figure 2, the greatest overall PE occurs for $\rho=0.1 \% \mathrm{w} / \mathrm{v}(\phi=7 \%)$. For low $\phi$, as shown in Figure 5(a)(i), the particles are well separated and the scattering cross-section can be approximated by (1) and (2). Further, since there is limited interaction among the particles, the total scattered flux will increase linearly with increasing $\phi$. However, at higher particle concentrations, particles tend to aggregate. This aggregation can be understood based on the dynamics of the spin-coating process. Spin-coating results in the formation of a thin layer of fluid in which particles are embedded. As the fluid film evaporates, attractive capillary forces can pull the particles closer to one another [29]. As the particle concentration increases, the initial interparticle separation in the fluid film decreases and within the time scale of drying of the fluid film, particles can attain maximal proximity, that is, the interparticle distance becomes close to particle diameter.

The optical properties of clusters and strings can be understood by examining the interparticle distances. When two metallic particles are within close proximity of each other, each individual particle unit forms a charge distribution of end-to-end dipoles [27, 28]. Linear chains of NPs act as a single dipole and the resultant effect is a red-shifting of the LSPR, which can be explained by the hybridization theory $[27,28]$. Specifically, the conducting electrons in the metal NPs may be modeled as a hybrid fluid form of electron density 


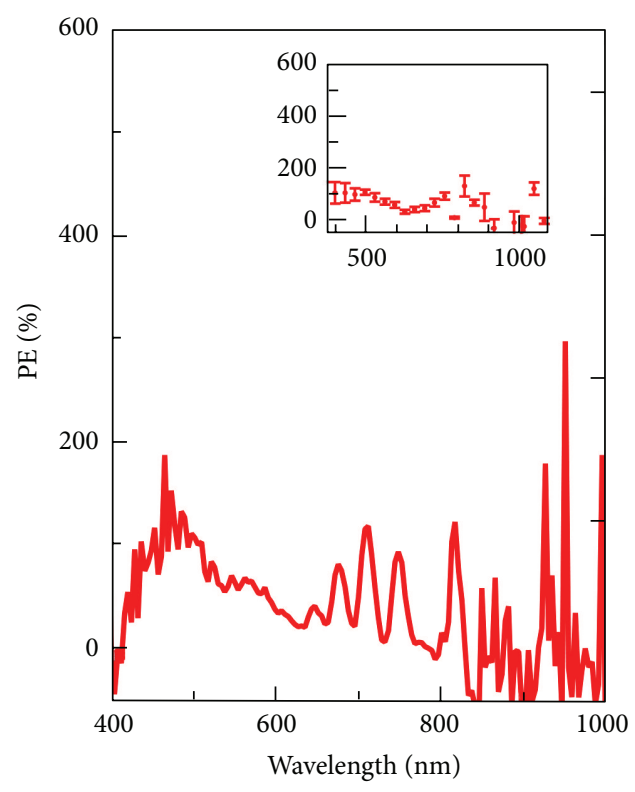

(a)

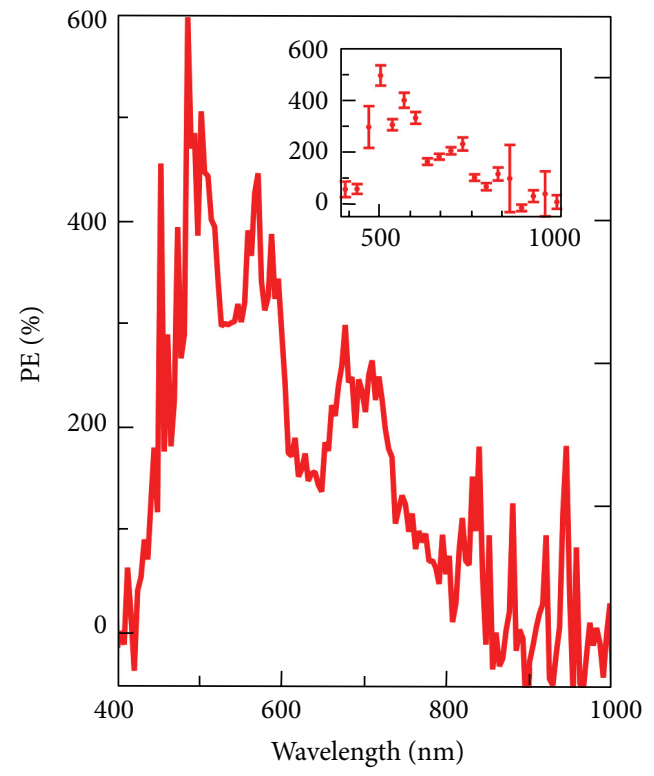

(b)

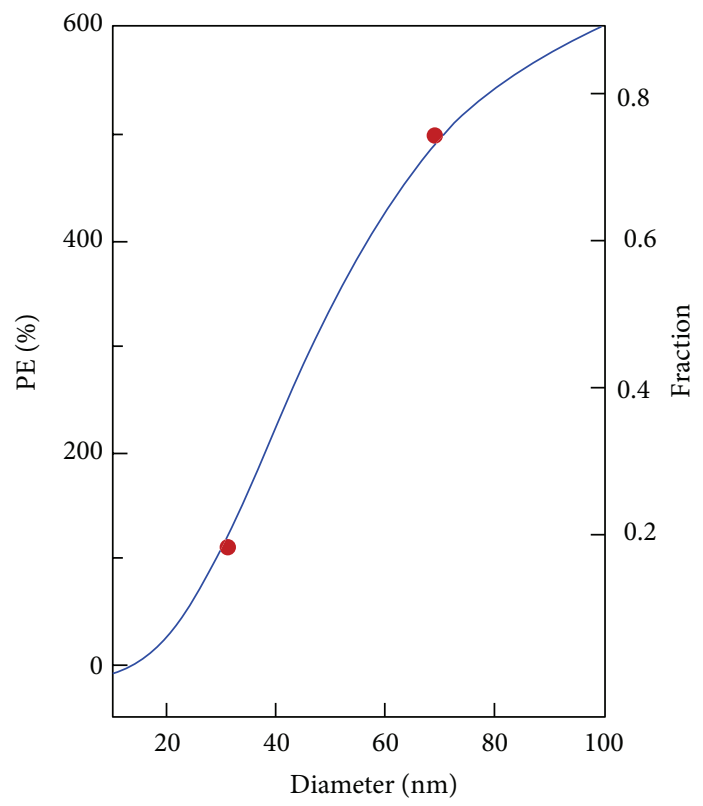

(c)

FIGURE 4: PE response of (a) $31 \mathrm{~nm}$ and (b) $69 \mathrm{~nm}$ synthesized silver particle coatings. Graphic insets of error bars show the standard error for the measurements. (c) Albedo of particles at $500 \mathrm{~nm}$ (blue line) and normalized PE responses for 31, 40, and $69 \mathrm{~nm}$ diameters (red circle).

with a unique dielectric constant. As the interparticle distance decreases, the hybridization effect and the red-shifting of the surface resonance plasmon mode become more pronounced.

When particles are not in a linear formation, the dipoles no longer align end-to-end. Instead, the interparticle interactions arise from multiple spatial directions and multipoles are induced within the individual particles. Multipoles are inefficient radiators, and have much lower absorption and scattering strengths compared to single particles $[27,30]$. Multipoles also induce a separate surface plasmon resonance peak that is different from the dipole peak, which becomes more distinct and disperse as more particles are added to the cluster. Clusters are extremely sensitive to light polarization and geometric arrangements. Super-radiant (dipole) and subradiant (multipole) modes form separate resonance peaks that quickly separate and diminish in intensity as the cluster gets larger because of destructive interference. The superradiant plasmon mode always red-shifts, while the subradiant modes either show a slight-blue shift or no shift at all $[27,28]$. This effect is seen in both aggregates of NPs in solution and also in large, extended close packed disordered and ordered NP interfaces [27]. 


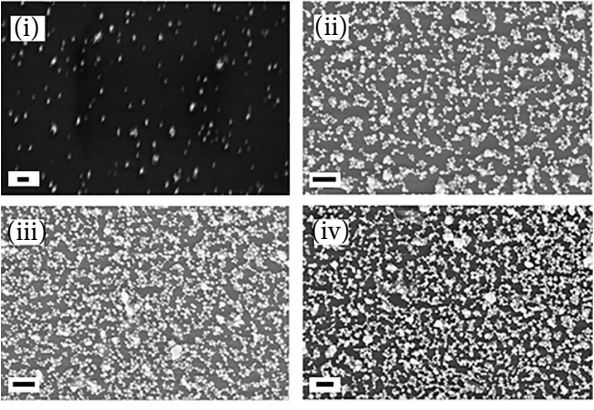

(a)

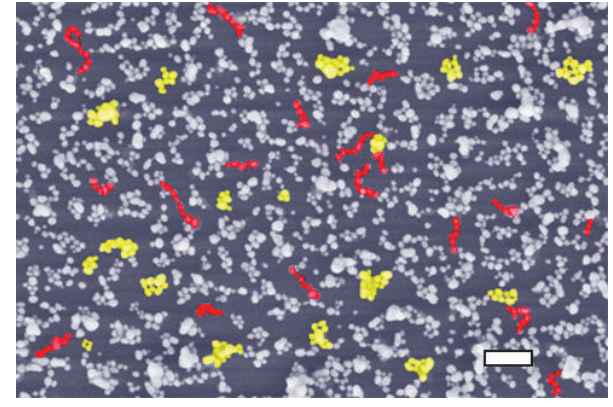

(b)

FIgURE 5: (a) SEM pictures of (i) $0.1 \%$, (ii) $1 \%$, (iii) $5 \%$, and (iv) $10 \%$ w/v solutions on SOI wafer. (b) SEM of $1 \%$ w/v Ag solution on SOI with example strings (red) and clusters (yellow) colorized. All scale bars are $200 \mathrm{~nm}$.

Particle aggregates on the samples can be seen in Figure 5(a)(ii)-(iv) for $\rho \geq 1 \%$. They are classified into strings (red) and clusters (yellow) and highlighted in Figure 5(b). A string is defined to be an approximately linear aggregate of three or more particles. A cluster is defined to be a non-linear aggregate of three or more particles where each particle has two or more neighbors within a single particle diameter. In Figure 2, the PE for $\rho=0.1 \% \mathrm{w} / \mathrm{v}$ shows a uniform broadband response. The only noticeable peaks that are observed in the $\mathrm{PE}$ spectrum are resonances from $\mathrm{Ag}$ in $\mathrm{SiO}_{2}$ and $\mathrm{Si}$. As the coatings become denser and particle aggregation occurs, the PE spectrum exhibits shifting of responses into the red band. The red-shifting narrows the overall bandwidth of $\mathrm{PE}$ since there is no absorbance above the bandgap wavelength of $\mathrm{c}-\mathrm{Si}$. The net result of the narrowing of the bandwidth is a reduction in PE. Irrespective of the morphology of the aggregates, that is, whether they are strings or clusters, they exhibit strong multipole characteristics, thus reducing the overall enhancement. As strings get longer and clusters get larger, the red-shifting becomes more pronounced as the absorption bandwidths narrow, with the $10 \% \mathrm{w} / \mathrm{v}$ solution producing the largest red-shift. Figure 6 shows $\phi$ as well as the number of strings and clusters as a function of $\rho$ in the feed solution. The surface coverage first increases linearly with increasing $\rho$ and appears to approach a plateau for higher $\rho$ possibly due to the formation of multilayered aggregates. The number of string and clusters grows linearly with increasing $\rho$ for $\rho \leq 1 \% \mathrm{w} / \mathrm{v}$. As $\rho$ is further increased, the rate of formation of aggregates decreases at the expense of having larger clusters and strings. This results in the saturation in the number of strings and clusters for $\rho$ between 5\% and 10\% solution $\mathrm{w} / \mathrm{v}$. The larger aggregates are detrimental to $\mathrm{PE}$ as seen in Figure 3.

The $31 \mathrm{~nm}$ glucose-capped particles showed a broadband enhancement with modest enhancement gains. The maximum enhancement gain using thermal evaporation to create particle sizes of $16 \mathrm{~nm}$ is $33 \%$, where the particles are closely packed Ag disks [14]. This shows that using chemical fabrication and the spin-coating technique produce superior PE results compared to the thermal evaporation method. Chemically synthesizing the NP to more desirable shapes such as a cylinder may produce an even greater PE

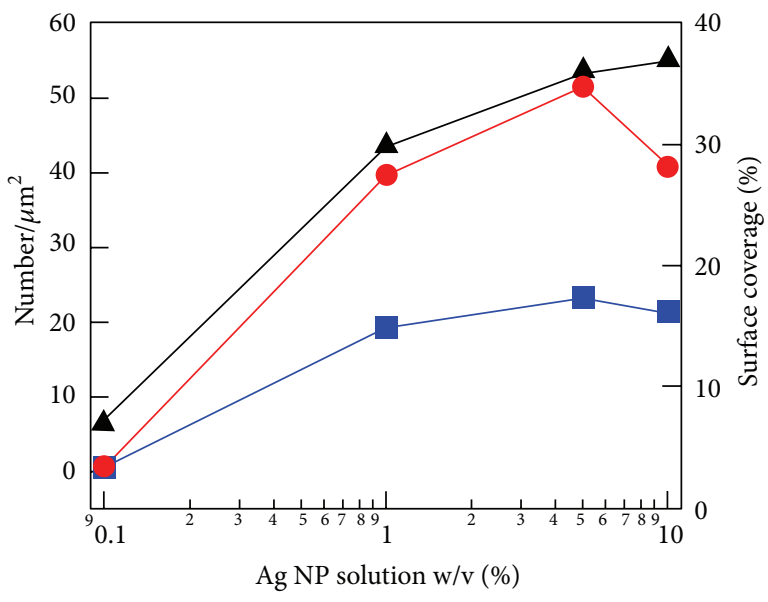

FIGURE 6: Particle surface coverage (black line) and the number of particle formations versus silver concentration in solution. The number of clusters (red line) and the number of strings (blue line) are shown per $\mu \mathrm{m}^{2}$.

response [8]. The $69 \mathrm{~nm}$ particles produced huge PE gains corresponding to the greater dipole strength. The increasing particle volume also causes red-shifts in the LSPR. As the particle size increases, multipole modes contribute to the absorption and scattering cross-section [31]. In Figure 4(b), four peak enhancements, namely, at $\approx 490,575,710$ and $830 \mathrm{~nm}$ range can be seen. The peaks at 490 and $830 \mathrm{~nm}$ can be attributed to the long range dipole-dipole interactions. Due to particle size, the long range interaction resonance peaks become slightly red-shifted as compared to their smaller diameter counterparts [26]. Additionally, the peaks occurring at $\approx 575$ and $710 \mathrm{~nm}$ are the signature of the $\mathrm{Ag}$ glucose core-shell architecture. Because of the relatively larger particle size compared to the visible wavelength range, (1) and (2) do not accurately capture the scattering and absorption cross-sections. Hence, Mie theory was used to calculate the scattering and absorption cross-sections of a $69 \mathrm{~nm} \mathrm{Ag}$ nanoparticle embedded in glucose [17, 32]. As shown in Figure 7, two surface plasmon resonance peaks are predicted at 560 and $730 \mathrm{~nm}$, corresponding to the dipole and multipole 


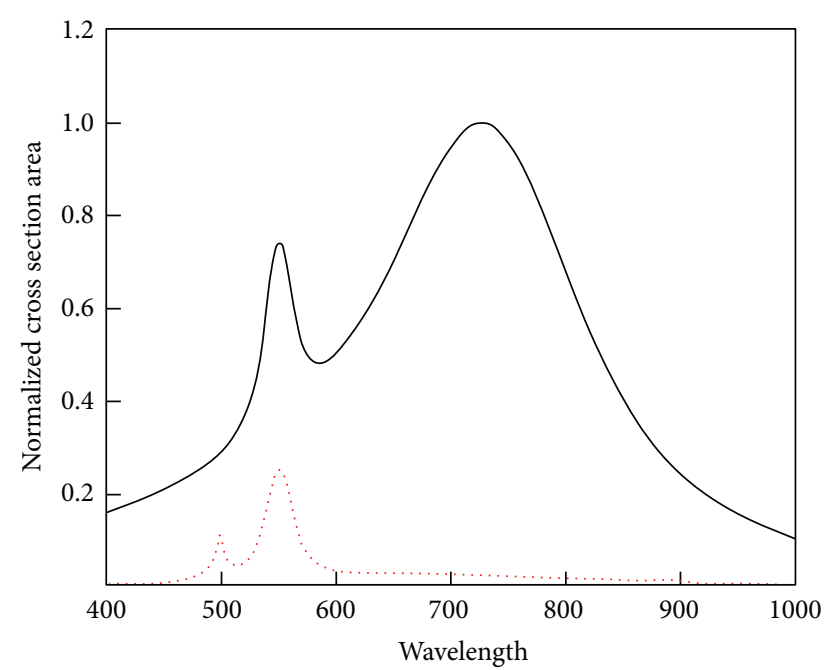

FIGURE 7: Mie theory predictions for the scattering (black) and absorption (red) cross-sectional areas normalized with respect to the maximum scattering cross-sectional area for a $69 \mathrm{~nm} \mathrm{Ag}$ particle embedded in glucose.

resonances respectively, consistent with the peaks in the measured photocurrent response.

\section{Conclusion}

Chemically synthesized silver nanoparticles have been spincoated onto a SOI device to fabricate light harvesting plasmonic interfaces, resulting in PE of up to $200 \%$. The correlation between $\mathrm{PE}$ and interface morphology has been identified. Specifically, we have shown the existence of an optimal window in the surface coverage of the particles $(\sim 7 \%)$ for which $\mathrm{PE}$ is maximally enhanced. This optimal range corresponds to an interface morphology that consists of wellseparated particles which can be treated as individual scattering elements. Hence, the PE increases approximately linearly with increasing $\phi$ until the optimal range is reached. In this regime the increases in $\mathrm{PE}$ and the scattering efficiency with increasing particle volume follow the same trend. Further increase in particle concentration beyond the optimal range results in particle aggregation into clusters and strings, both of which red-shift and narrows the PE spectrum, causing a decrease in the overall PE.

Our approach leverages the extensive knowledge base that exists on the chemical synthesis of NPs with admirable control on their size, shape and architecture [33]. Once NPs with tailored optical properties are synthesized, spincoating offers a cost-effective and robust means to transfer them from solution to the substrate. Evaporation of the solvent occurs at room temperature, thus obviating additional energy-intensive steps. This simple nanomanufacturing route to creating plasmonic interfaces on SOI devices presented in this paper paves way to future research to explore the effect of evanescent wave source propagation as the particle distance from the surface could be controlled through shell thickness. Finally, Ag NPs coatings can provide relatively inexpensive means to broadband light harvesting especially if the resulting PE is sufficiently large to offset the material $\left(\sim \$ 1 / \mathrm{m}^{2}\right)$ and manufacturing costs. This study clearly demonstrates that by tailoring the interface morphologies through optimizing particle and process parameters the PE could be tripled, a finding that motivates future translational research on integration of such plasmonic layers into thin film $\mathrm{Si}$ architectures.

\section{Conflict of Interests}

The authors declare that there is no conflict of interests regarding the publication of this paper.

\section{Acknowledgments}

The authors gratefully acknowledge financial support from the National Science Foundation through Grants CBET 1049454 and ICORPS 124248. The authors are thankful to Professor Eric Schiff and Mr. Hui Zhao for providing access to the photocurrent measurement facility, Professor Mathew Maye for the use of the DLS equipment and Professor Amit Agrawal for valuable discussions about this work and help in preparing Figure 1(a). The SOI device fabrication was performed at the Cornell NanoScale Facility, a member of the National Nanotechnology Infrastructure Network, which is supported by the National Science Foundation (Grant ECCS 0335765).

\section{References}

[1] A. G. Aberle, "Thin-film solar cells," Thin Solid Films, vol. 517, no. 17, pp. 4706-4710, 2009.

[2] G. Wilson, "Research Cell Efficiency Records," National Center for Photovoltaics, 2012, http://www.nrel.gov/ncpv/ images/efficiency_chart.jpg.

[3] K. R. Catchpole and A. Polman, "Plasmonic solar cells," Optics Express, vol. 16, no. 26, pp. 21793-21800, 2008.

[4] C. F. Bohren and D. R. Huffman, Absorption and Scattering of Light by Small Particles, John Wiley \& Sons, New York, NY, USA, 1983.

[5] W. Lukosz and R. E. Kunz, "Light emission by magnetic and electric dipoles close to a plane interface. I . Total radiated power," Journal of the Optical Society of America, vol. 67, no. 12, pp. 1607-1614, 1977.

[6] M. Kerker, W. Lukosz, and R. E. Kunz, "Light emission by magnetic and electric dipoles close to a plane dielectric interface. II. Radiation patterns of perpendicular oriented dipoles," Journal of the Optical Society of America, vol. 67, no. 12, pp. 1615-1619, 1977.

[7] L. Novotny and B. Hecht, Principles of Nano-Optics, Cambridge University Press, New York, NY, USA, 2006.

[8] H. A. Atwater and A. Polman, "Plasmonics for improved photovoltaic devices," Nature Materials, vol. 9, no. 3, pp. 205213, 2010.

[9] K. Nakayama, K. Tanabe, and H. A. Atwater, "Plasmonic nanoparticle enhanced light absorption in GaAs solar cells," Applied Physics Letters, vol. 93, no. 12, Article ID 121904, 2008.

[10] V. E. Ferry, M. A. Verschuuren, H. B. T. Li et al., "Light trapping in ultrathin plasmonic solar cells," Optics Express, vol. 18, no. 13, pp. A237-A245, 2010. 
[11] A. I. Kuznetsov, J. Koch, and B. N. Chichkov, "Nanostructuring of thin gold films by femtosecond lasers," Applied Physics A, vol. 94, no. 2, pp. 221-230, 2009.

[12] J. Trice, D. Thomas, C. Favazza, R. Sureshkumar, and R. Kalyanaraman, "Pulsed-laser-induced dewetting in nanoscopic metal films: theory and experiments," Physical Review B, vol. 75, no. 23, Article ID 235439, 2007.

[13] J. Trice, C. Favazza, D. Thomas, H. Garcia, R. Kalyanaraman, and R. Sureshkumar, "Novel self-organization mechanism in ultrathin liquid films: theory and experiment," Physical Review Letters, vol. 101, no. 1, Article ID 017802, 2008.

[14] S. Pillai, K. R. Catchpole, T. Trupke, and M. A. Green, "Surface plasmon enhanced silicon solar cells," Journal of Applied Physics, vol. 101, no. 9, Article ID 093105, 2007.

[15] H. R. Stuart and D. G. Hall, "Island size effects in nanoparticleenhanced photodetectors," Applied Physics Letters, vol. 73, no. 26, pp. 3815-3817, 1998.

[16] A. M. Eremenko, N. P. Smirnoval, I. P. Mukhal, and H. R. Yashan, "Silver and gold nanoparticles in silica matrices: synthesis, properties, and application," Theoretical and Experimental Chemistry, vol. 46, no. 2, pp. 65-88, 2010.

[17] K. L. Kelly, E. Coronado, L. L. Zhao, and G. C. Schatz, "The optical properties of metal nanoparticles: the influence of size, shape, and dielectric environment," Journal of Physical Chemistry B, vol. 107, no. 3, pp. 668-677, 2003.

[18] T. Cong, S. N. Wani, P. A. Paynter, and R. Sureshkumar, "Structure and optical properties of self-assembled multicomponent plasmonic nanogels," Applied Physics Letters, vol. 99, no. 4, Article ID 043112, 2011.

[19] D. Erickson, D. Sinton, and D. Psaltis, "Optofluidics for energy applications,” Nature Photonics, vol. 5, no. 10, pp. 583-590, 2011.

[20] A. Panacek, L. Kvítek, R. Prucek et al., "Silver colloid nanoparticles: synthesis, characterization, and their antibacterial activity," The Journal of Physical Chemistry B, vol. 110, no. 33, pp. 1624816253, 2006.

[21] A. W. Sarto and B. J. van Zeghbroeck, "Photocurrents in a metalsemiconductor-metal photodetector," IEEE Journal of Quantum Electronics, vol. 33, no. 12, pp. 2188-2194, 1997.

[22] E. Bassous, M. Scheuermann, V. P. Kesan, M. Ritter, J. M. Haibout, and S. S. Iyer, "A high-speed silicon metal-semiconductormetal photodetector fully integrable with (Bi)CMOS circuits," in International Electronic Devices Meeting, pp. 187-190, 1991.

[23] L. Colace, G. Masini, F. Galluzzi et al., "Metal-semiconductormetal near-infrared light detector based on epitaxial $\mathrm{Ge} / \mathrm{Si}$," Applied Physics Letters, vol. 72, no. 24, pp. 3175-3177, 1998.

[24] S. H. Lim, W. Mar, P. Matheu, D. Derkacs, and E. T. Yu, "Photocurrent spectroscopy of optical absorption enhancement in silicon photodiodes via scattering from surface plasmon polaritons in gold nanoparticles," Journal of Applied Physics, vol. 101, no. 10, Article ID 104309, 2007.

[25] H. R. Stuart and D. G. Hall, "Enhanced dipole-dipole interaction between elementary radiators near a surface," Physical Review Letters, vol. 80, no. 25, pp. 5663-5666, 1998.

[26] B. J. Soller and D. G. Hall, "Scattering enhancement from an array of interacting dipoles near a planar waveguide," Journal of the Optical Society of America B, vol. 19, no. 10, pp. 2437-2448, 2002.

[27] L. S. Slaughter, B. A. Willingham, W. S. Chang, M. H. Chester, N. Ogden, and S. Link, "Toward plasmonic polymers," Nano Letters, vol. 12, no. 8, pp. 3967-3972, 2012.
[28] N. J. Halas, S. Lal, W.-S. Chang, S. Link, and P. Nordlander, "Plasmons in strongly coupled metallic nanostructures," Chemical Reviews, vol. 111, no. 6, pp. 3913-3961, 2011.

[29] P. A. Kralchevsky and N. D. Denkov, "Capillary forces and structuring in layers of colloid particles," Current Opinion in Colloid and Interface Science, vol. 6, no. 4, pp. 383-401, 2001.

[30] J. B. Lassiter, H. Sobhani, J. A. Fan et al., "Fano resonances in plasmonic nanoclusters: geometrical and chemical tunability," Nano Letters, vol. 10, no. 8, pp. 3184-3189, 2010.

[31] S. Link and M. A. El-Sayed, "Size and temperature dependence of the plasmon absorption of colloidal gold nanoparticles," Journal of Physical Chemistry B, vol. 103, no. 21, pp. 4212-4217, 1999.

[32] K. Fuchs and U. Kaatze, "Molecular dynamics of carbohydrate aqueous solutions. dielectric relaxation as a function of glucose and fructose concentration," Journal of Physical Chemistry B, vol. 105, no. 10, pp. 2036-2042, 2001.

[33] L. M. Liz-Marzán, M. Giersig, and P. Mulvaney, "Synthesis of nanosized gold-silica core-shell particles," Langmuir, vol. 12, no. 18, pp. 4329-4335, 1996. 

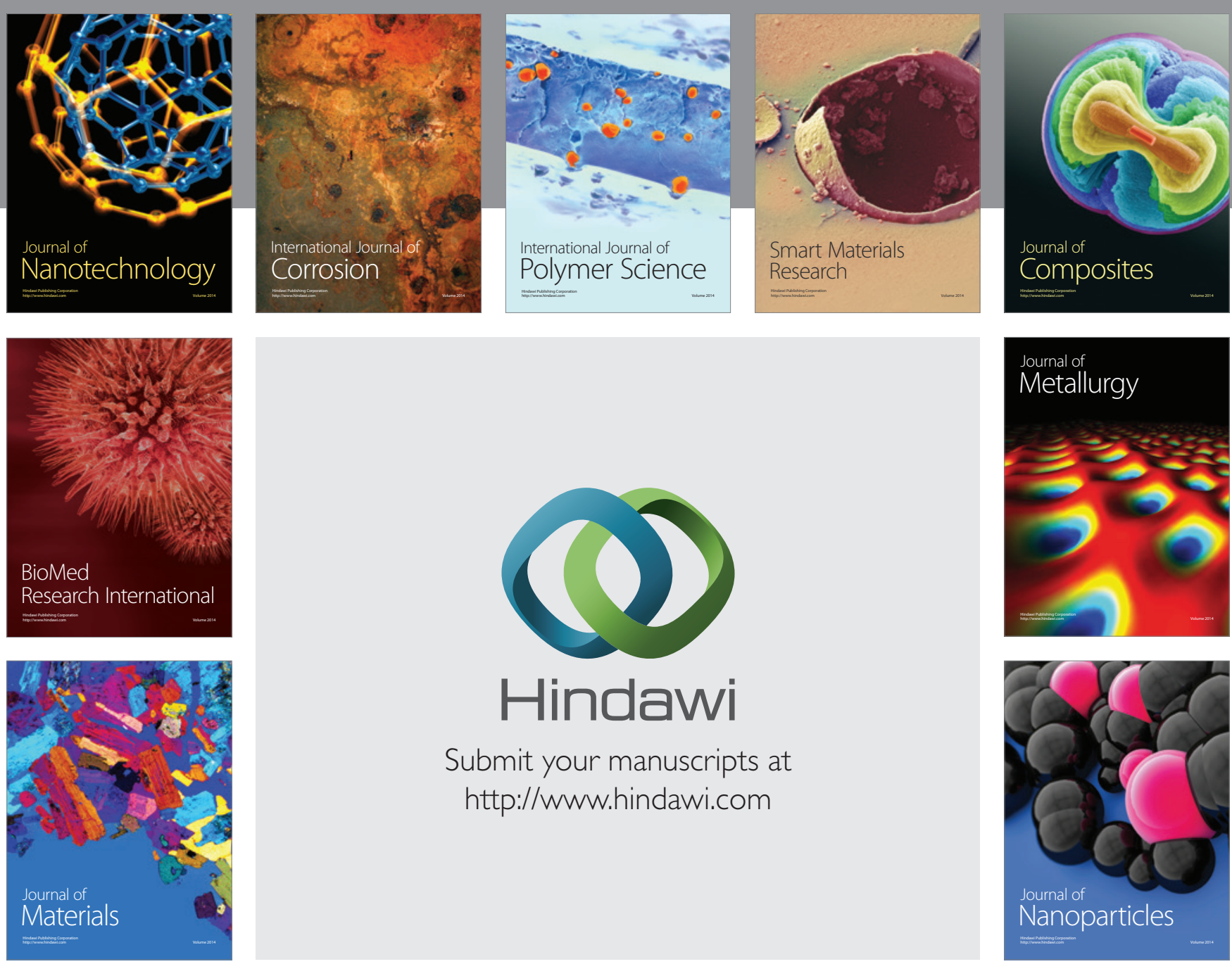

Submit your manuscripts at http://www.hindawi.com
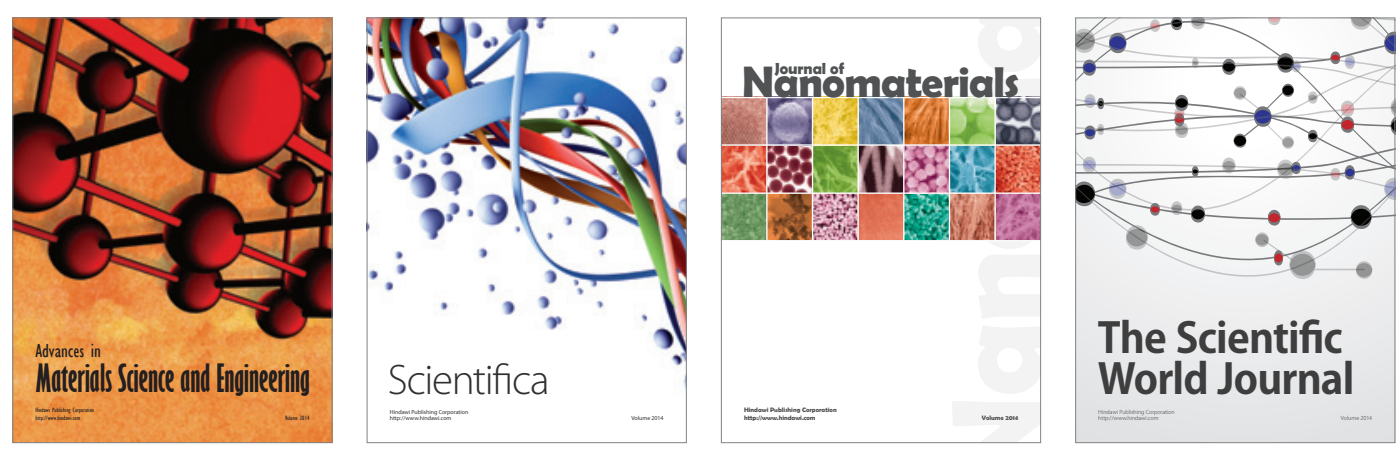

\section{The Scientific World Journal}
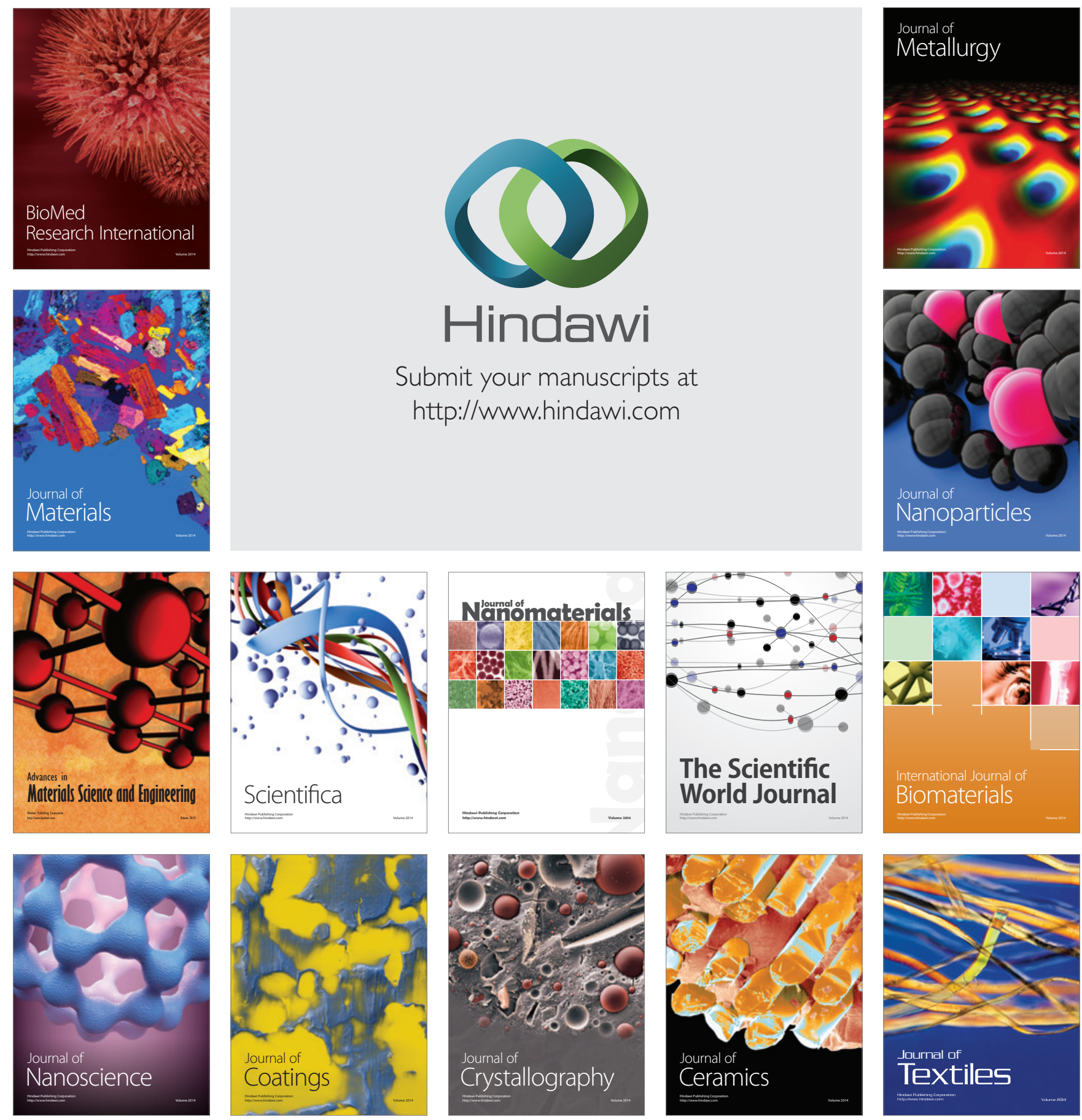